\title{
ARTICLE
}

\section{Properties of an energy degrader for light ions}

\author{
Frédéric Stichelbaut ${ }^{*}$ and Yves Jongen \\ Ion Beam Applications s.a., Chemin du Cyclotron, 3, B-1348 Louvain-la-Neuve, Belgium
}

\begin{abstract}
The IBA Company develops an energy selection system coupled to a superconducting cyclotron to generate light ion beams for hadron therapy with energy ranging from $100 \mathrm{MeV}$ to $400 \mathrm{MeV}$ per nucleon. Transmission properties of this energy selection system and fragmentation of the incident ion beams on the energy degrader are studied using the PHITS Monte Carlo simulation code.
\end{abstract}

\section{Keywords: energy degrader; hadron therapy; ion fragmentation; PHITS}

\section{Introduction}

The Ion Beam Applications (IBA) Company works on the development of a superconducting cyclotron able to deliver various light ion beams with a maximal energy of $400 \mathrm{MeV}$ per nucleon $(\mathrm{MeV} / \mathrm{u})$ [1]. This accelerator named C400 is designed to equip a hadrontherapy centre such as the ARCHADE project in France [2].

Fully stripped ions from ${ }^{4} \mathrm{He}$ up to ${ }^{16} \mathrm{O}$ can be accelerated inside the $\mathrm{C} 400$. They are extracted with an electromagnetic septum at fixed energy. To modulate the energy of the beams delivered to the patients, use is made of an energy selection system similar to the one developed by IBA for Proton Therapy (PT) systems. Figure 1 shows the layout of the Energy Selection System (ESS) for proton therapy system. After the beam extraction, a set of quadrupoles is used to focus the beam in order to obtain a minimal beam size at the entrance of the energy degrader. The later consists of a graphite wheel with variable thickness to degrade the energy beam from $230 \mathrm{MeV}$ down to a minimal energy of $70 \mathrm{MeV}$. Behind the degrader, a collimator is used to limit the emittance of the transmitted beam. After another set of quadrupoles to refocus the beam, two sets of 2 dipole magnets are installed to bend the beam towards the irradiation rooms. The first set of dipoles act as an energy analyzer and a momentum slit is placed in the middle of the two sets of dipoles to limit the energy spread of the transported beam to a maximal value $\Delta \mathrm{E} / \mathrm{E}=0.6 \%$.

A similar ESS is under design to transport ion beams extracted from the C400. Compared to protons, light ions exhibit less angular divergence because of their higher masses but suffer from ion fragmentation due to nucleus-nucleus interactions inside the degrader, leading to a smaller transmission efficiency through the degrader. Charged ion fragments with the same magnetic rigidity as

*Corresponding author. Email: frederic.stichelbaut@iba-group.com the primary beam can also be transported by the beam line and contaminate the beam reaching the patient. The goal of this study is to compare this contamination with the ion fragmentation occurring in the patient itself.

Large fluxes of secondary neutrons and photons are also produced by the degrader but they will flight in stray direction and will not be transported to the patient rooms. Shielding is designed to reduce their contribution to annual radiation doses below the dose limit applicable to a public area. Ion fragmentation will also occur at the level of the momentum slit but beam losses are strongly reduced compared to those observed at the degrader level and this contamination will be neglected here.

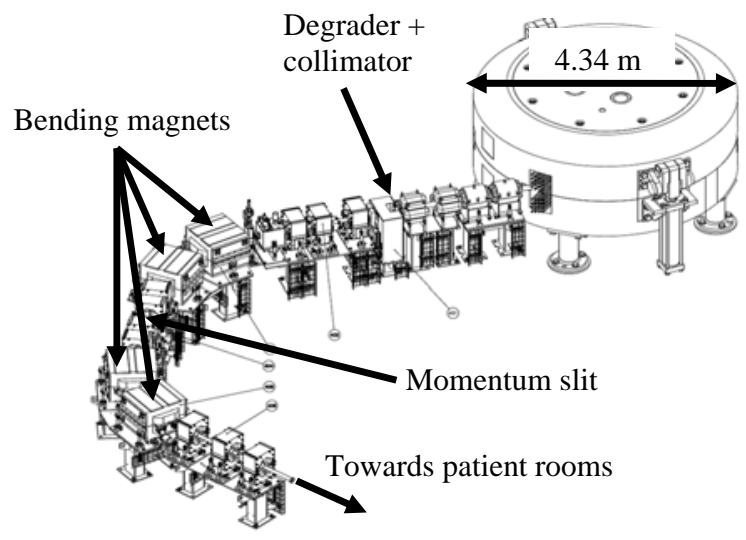

Figure 1. Layout of the energy selection system.

The ESS transmission efficiency has been estimated for all possible ion species using the PHITS Monte Carlo simulation code and is presented in section 2 [3].

The ion fragment production by the ESS has been analysed for ${ }^{12} \mathrm{C}$ ion beams only and is the subject of section 3 . The carbon ion beam fragmentation inside the patient is studied in section 4 and the fragment yields are compared to those produced by the ESS. 


\section{ESS transmission properties}

Transmission properties of the ESS are studied using the Particle and Heavy-Ion Transport code System, PHITS version 2.24 [3]. Ions ${ }^{4} \mathrm{He},{ }^{6} \mathrm{Li},{ }^{10} \mathrm{~B},{ }^{12} \mathrm{C},{ }^{14} \mathrm{~N}$ and ${ }^{16} \mathrm{O}$ can be accelerated by the $\mathrm{C} 400$ and have been considered in this study. Nucleus-nucleus interactions are simulated using the JAERI Quantum Molecular Dynamics model (JQMD) [4]. The evaporation and fission processes are simulated with the Generalized Evaporation Model (GEM). To handle the energy loss, Coulomb diffusion and energy straggling processes, use is made of the ATIMA code implemented in PHITS. Finally, the total reaction cross section for heavy-ion collisions is parameterized using the Tripathi formula [5].

The major element of the ESS is the energy degrader. It is usually made of high-density graphite or beryllium to limit the Coulomb diffusion and both elements have been studied. Nuclear interactions inside that degrader lead to a significant reduction of the transmitted beam intensity. As shown in Figure 2 for $400 \mathrm{MeV} / \mathrm{u}$ ions impinging on a graphite degrader, the transmission efficiency varies with the transmitted energy and the ion mass. The transmitted energy considered here is the average ion energy obtained by fitting the energy distribution to a Gaussian function. For light ions and low transmitted energy, the beam losses can be as high as $80 \%$ of the incident beam.

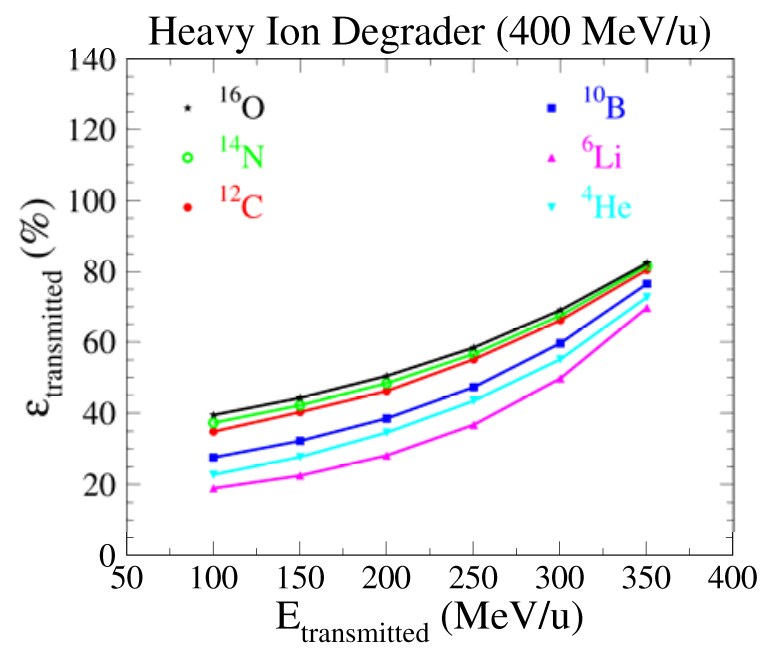

Figure 2. Transmission efficiency of a graphite degrader for light ions.

After the energy degrader, the ion beam must pass through a Ta collimator in order to limit the beam divergence to less than $12 \mathrm{mrad}$. The angular distribution of the transmitted ions is computed by measuring the forward current crossing the exit surface of the degrader ([T-Cross]-type tally) and the selection efficiency of the angular cut is determined.

The beam must also pass through energy slits to reduce the beam energy spread to $\Delta \mathrm{E} / \mathrm{E}<0.6 \%$. To take into account correlations between the transmitted polar angle and energy, this energy cut is only applied to ions selected by the angular cut.

The ESS transmission efficiency is obtained by combining the degrader transmission efficiency with the efficiencies of the angular and energy cuts. Its evolution with the transmitted beam energy is shown in Figure 3 for all studied ion species. For the lowest energy of 100 $\mathrm{MeV} / \mathrm{u}$, this efficiency remains larger than $1 \%$ for the ${ }^{10} \mathrm{~B}$ and heavier ions but becomes smaller than $1 \%$ for ${ }^{6} \mathrm{Li}$ and ${ }^{4} \mathrm{He}$ ions.

The analysis was repeated for ${ }^{12} \mathrm{C}$ ion beams using a beryllium degrader. The lower atomic number of beryllium compared to graphite allows a reduction of the beam divergence after the degrader. However, the transmission efficiency of ${ }^{12} \mathrm{C}$ ions is significantly reduced with beryllium, resulting in a decrease of the ESS efficiency at all transmitted energies when replacing graphite by beryllium.

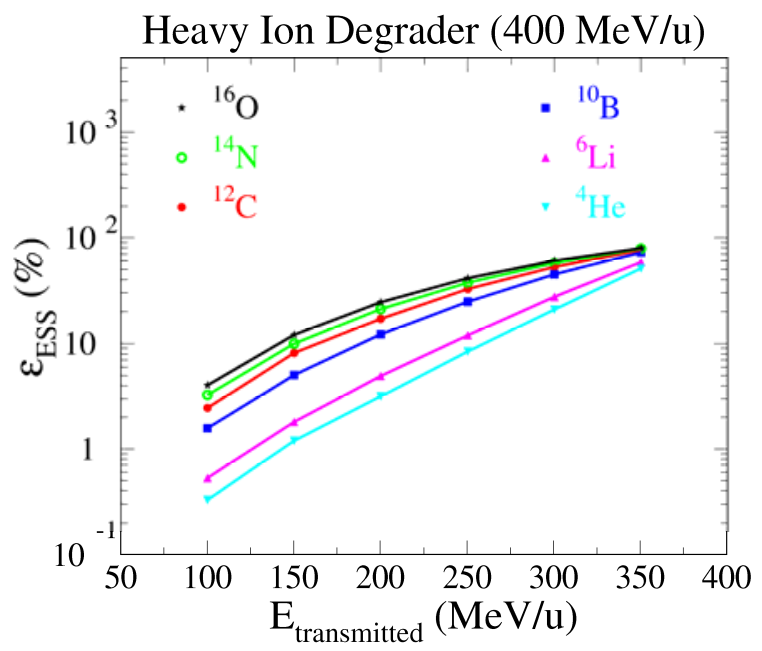

Figure 3. Transmission efficiency of the ESS with a graphite degrader for light ions from ${ }^{4} \mathrm{He}$ to ${ }^{16} \mathrm{O}$.

The ESS efficiency is an important aspect of the radiation shielding design for the cyclotron room of an hadron therapy centre [6]. To compensate for the beam losses inside the ESS, the beam intensity extracted from the cyclotron must be increased to guarantee the beam intensity required by the therapeutic treatments. A very low ESS efficiency would result in the need of very thick shielding walls around the cyclotron and the ESS. Based upon IBA experience in PT, a value of $1 \%$ for the ESS efficiency is fully acceptable.

\section{Ion fragmentation in degrader}

The nucleus-nucleus interactions between the primary ion beam and the degrader lead to a reduction of the beam transmission efficiency but also to the production of ion fragments polluting the therapeutic beam. Fragments with the same ratio charge/mass as the primary beam have the same magnetic rigidity and will be transported by the beam line towards the patient.

The fragment production by the degrader is studied with PHITS [3] using $400 \mathrm{MeV} / \mathrm{u}{ }^{12} \mathrm{C}$ ions impinging on a cylindrical graphite degrader with a radius of $10 \mathrm{~cm}$ and a variable thickness to select the transmitted beam energy. 
The incident beam is taken as point-like, mono-energetic and with no angular divergence. A [T-Cross] tally on the degrader exit surface allows the determination of the currents of the fragments escaping the degrader. Figure 4 shows the 15 largest yields obtained for a transmitted energy of $100 \mathrm{MeV} / \mathrm{u}$. The two largest yields correspond to protons and $\alpha$ particles, with values of 2.15 and 0.44 , respectively [7]. The large emission of $\alpha$ particles is explained by the important cross section for the fragmentation of ${ }^{12} \mathrm{C}$ ion into $3 \alpha$ 's.

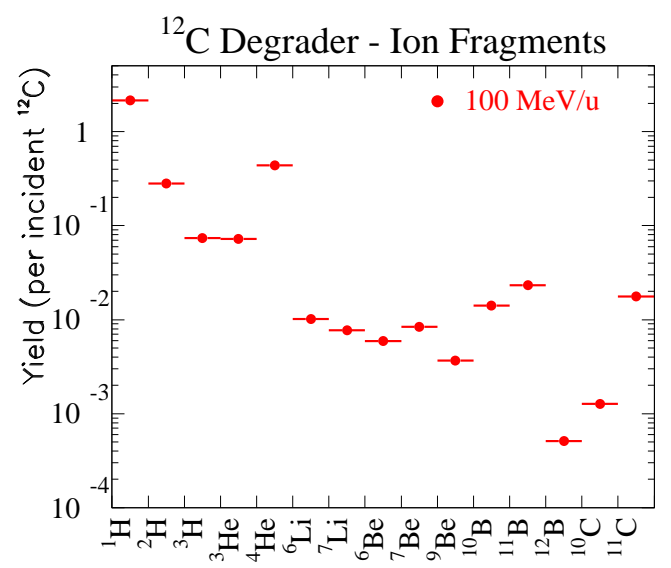

Figure 4. Production yields of escaping ion fragments for 400 $\mathrm{MeV} / \mathrm{u}{ }^{12} \mathrm{C}$ ions impinging on the degrader. The degrader thickness corresponds to transmitted energy of $100 \mathrm{MeV} / \mathrm{u}$.

Kinematic properties of the fragments are determined using a [T-Cross] tally on the degrader exit surface. As these fragments are produced by nucleus-nucleus interactions, they exhibit much broader energy and angular spectra than the transmitted ${ }^{12} \mathrm{C}$ ions. This is demonstrated in Figure $\mathbf{5}$ comparing the angular distributions of ${ }^{12} \mathrm{C}$ primary ions and various ion fragments. Even for moderate energy degradation to 300 $\mathrm{MeV} / \mathrm{u}$, light fragments are produced with very broad angular distributions, completely different from the transmitted ${ }^{12} \mathrm{C}$ ions. These distributions become even broader when the transmitted energy decreases.

Therefore, the collimator located downstream of the degrader will intercept most of these fragments, letting pass only those with a polar angle smaller than $12 \mathrm{mrad}$. Figure 6 shows that this simple angular acceptance cut reduces the transmitted yields by 2 to 3 orders of magnitude. Results presented in Figure 6 correspond to a transmission energy of $100 \mathrm{MeV} / \mathrm{u}$ and are also normalized per incident ${ }^{12} \mathrm{C}$ ion. After the angular cut, the largest fragment yield is reduced to $0.0076 \alpha$ particle per incident ${ }^{12} \mathrm{C}$.

\section{Ion fragmentation in patient}

Ion beams used to treat patients will also lead to the production of ion fragments inside the patient itself. It is thus interesting to compare these fragment production yields with those coming from the ESS.

The beam fragmentation in patient is studied for ${ }^{12} \mathrm{C}$ ion

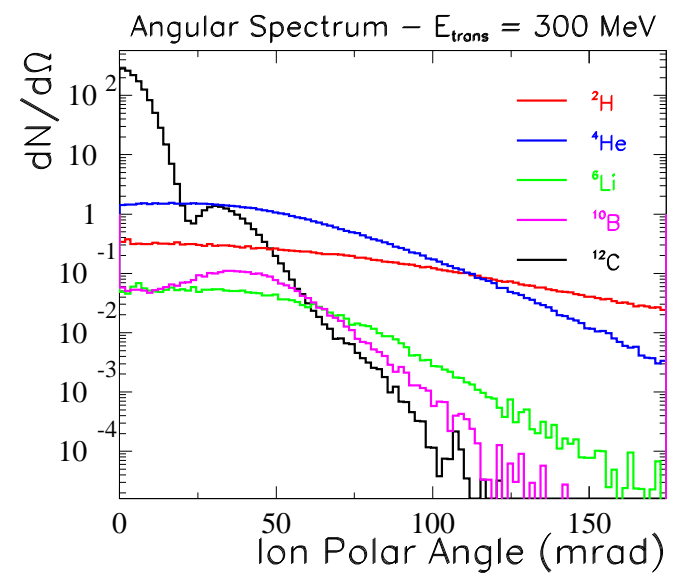

Figure 5. Angular distributions of ${ }^{12} \mathrm{C}$ primary ions and various ion fragments after the degrader. The primary beam consists of $400 \mathrm{MeV} / \mathrm{u}{ }^{12} \mathrm{C}$ ions and is transmitted with an energy of 300 $\mathrm{MeV} / \mathrm{u}$.

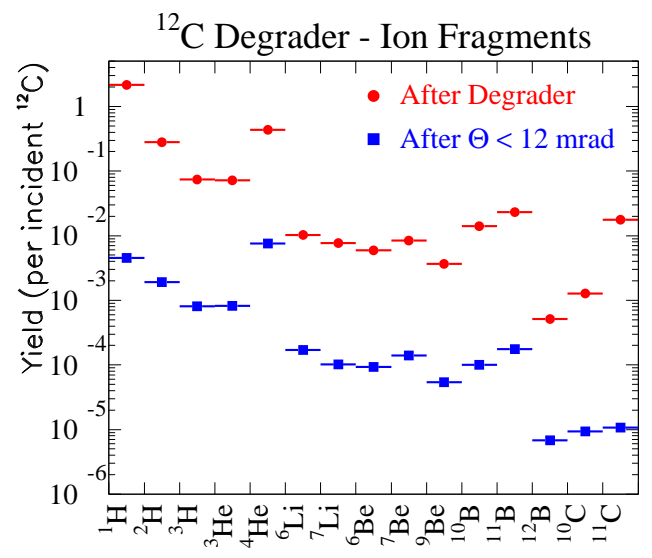

Figure 6. Comparison of the ion fragment yields obtained before and after applying the polar angle selection cut.

beams with energies ranging from $100 \mathrm{MeV} / \mathrm{u}$ up to 400 $\mathrm{MeV} / \mathrm{u}$. The primary beam impinges on a cylindrical water target with a length of $30 \mathrm{~cm}$ and a radius of $10 \mathrm{~cm}$. The irradiation field covers a $10 \times 10 \mathrm{~cm}^{2}$ surface and the beam is considered to be mono-energetic.

The evolution of ion fragment currents as a function of target depth is computed using a [T-Cross] tally on the same surface as the irradiation field. The forward current is computed every $\mathrm{cm}$ from the target entrance to the target exit and multiplied by the crossed surface to obtain the integrated current per incident ${ }^{12} \mathrm{C}$ ion.

The currents are computed for the same ion species as in the previous section. Figure 7 shows the evolution with depth of the ${ }^{4} \mathrm{He}$ currents for various incident beam energies. At the target entrance, the fragment yields increase almost linearly with depth and do not strongly depend upon the incident beam energy. These yields can be compared to the maximal yield coming from the ESS and represented by the horizontal dashed line in Figure 7. The ESS yield corresponds to transmitted beam energy of $100 \mathrm{MeV} / \mathrm{u}$ as this is the transmission energy leading to the largest fragment yields. It is clear that the ESS yield remains small compared to the patient yields. The comparison between ESS yields and patient yields 
obtained for beams of $100 \mathrm{MeV} / \mathrm{u}$ is shown in Figure 8 for the 15 possible fragments. These yields are normalized per transmitted ${ }^{12} \mathrm{C}$ ion. In all cases except for ${ }^{6} \mathrm{Be}$, the ESS yields are smaller than the patient yields obtained at a depth of $2 \mathrm{~cm}$.

The dose-depth distributions obtained in water for 100 $\mathrm{MeV} / \mathrm{u}{ }^{12} \mathrm{C}$ ions and the ${ }^{1} \mathrm{H}$ or ${ }^{4} \mathrm{He}$ fragments coming from the ESS are compared in Figure 9. Before the Bragg peak, the dose from ${ }^{4} \mathrm{He}$ represents less than $1 \%$ of the dose due to the ${ }^{12} \mathrm{C}$ ions. This fraction increases beyond the peak but the dose contribution is very small, of the order of $0.03 \mathrm{~Gy}$ per $\mathrm{nC}$ of ${ }^{12} \mathrm{C}$ beam reaching the patient. The contribution from protons is a factor 10 smaller. The dose distributions from these fragments do not exhibit a Bragg peak because these fragments possess a broad energy spectrum and not a well-defined energy value. The contribution from the other fragments coming from the ESS to the total dose is also extremely small.

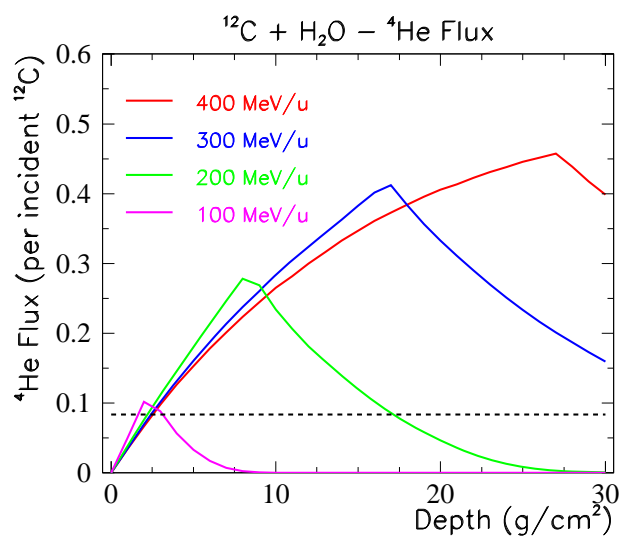

Figure 7. Evolution with depth of the generation of ${ }^{4} \mathrm{He}$ fragments for ${ }^{12} \mathrm{C}$ primary ions impinging on a water phantom. The dashed line shows the yield of ${ }^{4} \mathrm{He}$ fragments coming from the degrader for a transmission energy of $100 \mathrm{MeV} / \mathrm{u}$.

\section{Conclusions}

The Monte Carlo simulation of the light ions transmission through the energy selection system indicates that the transmission efficiency at the lowest energy of $100 \mathrm{MeV} / \mathrm{u}$ remains larger than $1 \%$ for most of the ion beams at the exception of ${ }^{4} \mathrm{He}$ and ${ }^{6} \mathrm{Li}$. This $1 \%$ efficiency is similar to the proton beam efficiency and is enough to ensure beam transport towards the irradiation rooms.

Our simulations also demonstrate that the ion fragments coming from the degrader will be efficiently suppressed by the ESS and will not significantly contribute to the patient dose.

These results confirm the possibility to use a fixed-energy cyclotron coupled to an energy degrader for a light ion therapy centre.

\section{References}

[1] Y. Jongen, IBA C400 Cyclotron project for hadron

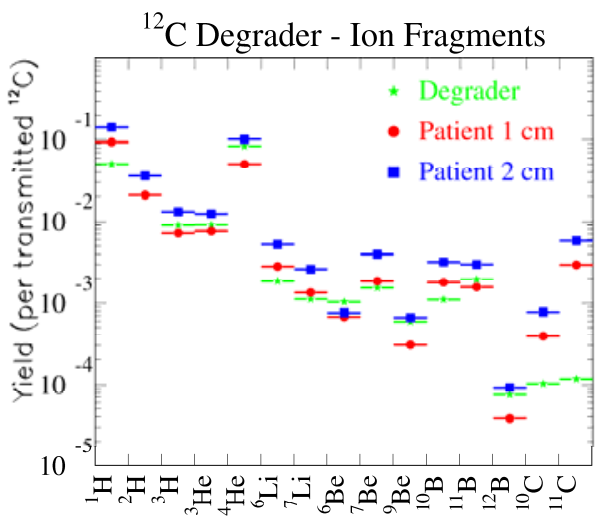

Figure 8. Comparison of the fragment yields generated by the ESS and those generated by the nuclear interactions inside the patient.

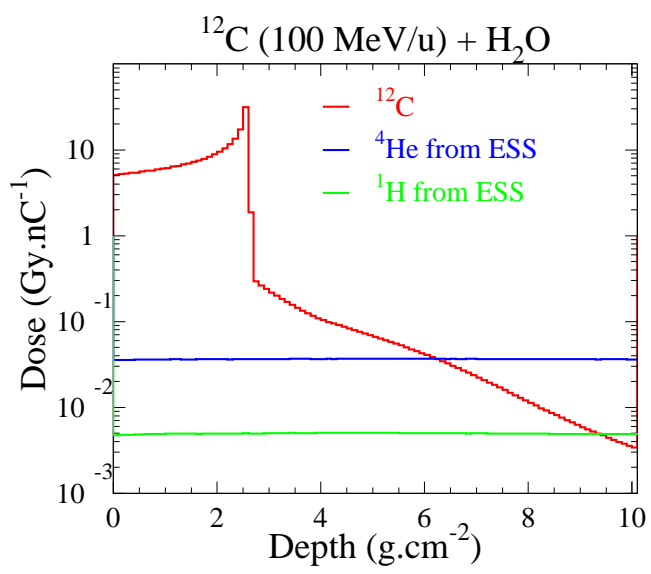

Figure 9. Dose-depth distributions for $100 \mathrm{MeV} / \mathrm{u}{ }^{12} \mathrm{C}$ ions in water and for the ${ }^{1} \mathrm{H}$ and ${ }^{4} \mathrm{He}$ ions coming from the ESS.

therapy, cyclotrons 2007, Giardini Naxos, Italy, (2007).

[2] F. Stichelbaut and Y. Jongen, Radioprotection studies for the ARCHADE carbon therapy centre, These proceedings.

[3] K. Niita, T. Sato, H. Iwase, H. Nose, H. Nakashima and L. Sihver, PHITS - A Particle and Heavy Ion Transport code System, Radiat. Meas. 41 (2006), p. 1080.

[4] K. Niita, S. Chiba, T. Maruyama, H. Takada, T. Fukahori, Y. Nakahara and A. Iwamoto, Analysis of the $(\mathrm{N}, \mathrm{xN}$ ') reactions by quantum molecular dynamics plus statistical decay model, Phys. Rev. C52 (1995), p. 2620.

[5] R. K. Tripathi, F. A. Cucinotta and J. W. Wilson, Accurate universal parametrization of absorption cross sections, Nucl. Instr. and Meth. B117 (1996), p. 347.

[6] F. Stichelbaut, T. Canon and Y. Jongen, Shielding studies for a hadron therapy centre. Nucl. Technol. 168 (2009), pp. 477-481.

[7]F. Stichelbaut and Y. Jongen, Shielding design for ETOILE hadron therapy centre, SATIF-10, Workshop Proceedings, Geneva, Switzerland, June 2010, OECD/NEA Publishing, (2011), p. 355. 